\title{
Homogeneous Riemannian Structures on Berger 3-Spheres *
}

\author{
P.M. Gadea And J.A. OubiñA ${ }^{\dagger}$
}

\begin{abstract}
The homogeneous Riemannian structures on the 3-dimensional Berger spheres, their corresponding reductive decompositions and the associated groups of isometries are obtained. The Berger 3-spheres are also considered as homogeneous almost contact metric manifolds.
\end{abstract}

\section{Introduction}

The Berger spheres $\mathbb{S}_{\varepsilon}^{3}$ are homogeneous Riemannian spaces diffeomorphic to the 3dimensional sphere. These spaces, found by M. Berger [3] in his classification of all simply connected normal homogeneous Riemannian manifolds of positive sectional curvature, have not constant curvature, and their metrics are obtained from the round metric on $\mathbb{S}^{3}$ by deforming it along the fibers of the Hopf fibration $\mathbb{S}^{3} \rightarrow \mathbb{S}^{2}$ by $\varepsilon$. These spaces are of great interest in Riemannian geometry and provide nice examples; for instance, they served as counterexamples to a conjecture of Klingenberg about closed geodesics (see, e.g., $[14$, p. 160]) and to conjectures on the first eigenvalue of the Laplacian on spheres $([2],[16])$.

On the other hand, it is well known the É. Cartan's classical characterization of Riemannian symmetric spaces as the spaces of parallel curvature (under certain conditions). This characterization was extended by Ambrose and Singer [1] to the homogeneous Riemannian case; they proved that a connected, simply connected and complete Riemannian manifold $(M, g)$ is homogeneous if and only if there exists a $(1,2)$ tensor field $S$ on $M$ (called by Tricerri and Vanhecke in [15] a homogeneous Riemannian structure) satisfying certain properties. Moreover, a homogeneous almost contact metric manifold $(M, \varphi, \xi, \eta, g)$ is called homogeneous if there exists a connected Lie group $G$ acting transitively on $(M, g)$ as a group of isometries which leave the almost contact metric structure $(\varphi, \xi, \eta)$ invariant; a homogeneous Riemannian structure satisfying an additional condition characterizes such a property.

The purpose of the present paper is to study the homogeneous Riemannian structures on the Berger 3-spheres. After some preliminaries ( $(2)$, we obtain all the homogeneous

*2000 Mathematics Subject Classification: 53C30, 53C25. Key Words: Berger spheres, homogeneous Riemannian structures, almost contact metric structures.

${ }^{\dagger}$ Partially supported by DGICYT, Spain, under grant BFM2002-00141 and by Xunta de Galicia under grant PGIDT01PXI20704PR. 
Riemannian structures on the Berger spheres (§3). These structures define Lie algebras with reductive decompositions, which have associated Lie groups with isometric actions on the spheres $(\S 4)$. Finally, in $\S 5$, we consider natural almost contact metric structures on the Berger spheres $\mathbb{S}_{\varepsilon}^{3}$, which are $\alpha$-Sasakian [9], $\alpha=\sqrt{\varepsilon}$, and become these manifolds into homogeneous almost contact metric manifolds.

\section{Preliminaries}

\subsection{Homogeneous Riemannian structures}

Let $(M, g)$ be a connected Riemannian manifold. Let $\nabla$ be the Levi-Civita connection of $g$ and $R$ its curvature tensor field, for which we adopt the conventions

$$
R_{X Y} Z=\nabla_{[X, Y]} Z-\nabla_{X} \nabla_{Y} Z+\nabla_{Y} \nabla_{X} Z, \quad R_{X Y Z W}=g\left(R_{X Y} Z, W\right),
$$

for all vector fields $X, Y, Z, W$ on $M$. A homogeneous Riemannian structure on $(M, g)$ is ([15]) a tensor field $S$ of type $(1,2)$ on $M$ such that the connection $\widetilde{\nabla}=\nabla-S$ satisfies

$$
\widetilde{\nabla} g=0, \quad \widetilde{\nabla} R=0, \quad \widetilde{\nabla} S=0 .
$$

We also denote by $S$ the associated tensor field of type $(0,3)$ on $M$ defined by $S_{X Y Z}=$ $g\left(S_{X} Y, Z\right)$.

Suppose that $(M, g)$ is a homogeneous Riemannian manifold, that is $M=G / H$, where $G$ is a connected Lie group acting transitively and effectively on $M$ as a group of isometries and $H$ is the isotropy group at a point $o \in M$. Then the Lie algebra $\mathfrak{g}$ of $G$ may be decomposed into a vector space direct sum $\mathfrak{g}=\mathfrak{h} \oplus \mathfrak{m}$, where $\mathfrak{h}$ is the Lie algebra of $H$ and $\mathfrak{m}$ is an $\operatorname{Ad}(H)$-invariant subspace of $\mathfrak{g}$. If $H$ is connected, the invariance condition $\operatorname{Ad}(H) \mathfrak{m} \subset \mathfrak{m}$ is equivalent to $[\mathfrak{h}, \mathfrak{m}] \subset \mathfrak{m}$. The vector space $\mathfrak{m}$ is identified with $T_{o}(M)$ through the isomorphism

$$
\begin{aligned}
\mu: \mathfrak{m} & \longrightarrow T_{o}(M) \\
X & \longmapsto X_{o}^{*},
\end{aligned}
$$

where $X^{*}$ is the Killing vector field generated on $M$ by the one-parameter subgroup $\{\exp s X\}$ of $G$ acting on $M$. Then, the canonical connection $\widetilde{\nabla}$ of $M=G / H$ (with regard to the reductive decomposition $\mathfrak{g}=\mathfrak{h} \oplus \mathfrak{m}$ ) is determined by its value at $o$, by

$$
\left(\widetilde{\nabla}_{X^{*}} Y^{*}\right)_{o}=-\left([X, Y]_{\mathfrak{m}}\right)_{o}^{*}, \quad X, Y \in \mathfrak{m}
$$

and $S=\nabla-\widetilde{\nabla}$ is the homogeneous Riemannian structure associated to the reductive decomposition $\mathfrak{g}=\mathfrak{h} \oplus \mathfrak{m}$.

Conversely, consider a homogeneous Riemannian structure $S$ on a connected, simply connected and complete Riemannian manifold $(M, g)$, fix a point $o \in M$ and put $\widetilde{\mathfrak{m}}=$ $T_{o}(M)$. If $\widetilde{R}$ is the curvature tensor of the connection $\widetilde{\nabla}=\nabla-S$, the holonomy algebra $\tilde{\mathfrak{h}}$ of $\widetilde{\nabla}$ is the Lie subalgebra (of the Lie algebra of antisymmetric endomorphisms of $\left(\widetilde{\mathfrak{m}}, g_{o}\right)$ ) 
generated by the operators $\widetilde{R}_{X Y}$, where $X, Y \in \widetilde{\mathfrak{m}}$. Then, a Lie bracket is defined in the vector space direct sum $\tilde{\mathfrak{g}}=\tilde{\mathfrak{h}} \oplus \widetilde{\mathfrak{m}}$ by

$$
\begin{array}{llrl}
{[U, V]} & =U V-V U, & & U, V \in \tilde{\mathfrak{h}}, \\
{[U, X]} & =U(X), & & U \in \tilde{\mathfrak{h}}, X \in \widetilde{\mathfrak{m}}, \\
{[X, Y]} & =\widetilde{R}_{X Y}+S_{X} Y-S_{Y} X, & & X, Y \in \widetilde{\mathfrak{m}},
\end{array}
$$

and $(\tilde{\mathfrak{g}}, \tilde{\mathfrak{h}})$ is the reductive pair associated to the homogeneous Riemannian structure $S$. Let $\widetilde{G}$ be the connected simply connected Lie group whose Lie algebra is $\tilde{\mathfrak{g}}$ and $\widetilde{H}$ the connected Lie subgroup of $\widetilde{G}$ whose Lie algebra is $\tilde{\mathfrak{h}}$. Then $\widetilde{G}$ acts transitively by isometries on $M$ and $M$ is diffeomorphic to $\widetilde{G} / \widetilde{H}$. If $\Gamma$ is the set of the elements of $\widetilde{G}$ which act trivially on $M$, then $\Gamma$ is a discrete normal subgroup of $\widetilde{G}$, and the Lie group $G=\widetilde{G} / \Gamma$ acts transitively and effectively on $M$ as a group of isometries, with isotropy group $H=\widetilde{H} / \Gamma$. Then $M$ is diffeomorphic to the homogeneous Riemannian manifold $G / H$.

\section{$2.2 \quad$ The Berger spheres}

As usual, we identify the sphere $\mathbb{S}^{3}$ and the Lie group $S U(2)$ by the map that sends $(z, w) \in \mathbb{S}^{3} \subset \mathbb{C}^{2}$ to $\left(\begin{array}{cc}z & w \\ -\bar{w} & \bar{z}\end{array}\right) \in S U(2)$. We consider the basis $\left\{X_{1}, X_{2}, X_{3}\right\}$ of the Lie algebra $\mathfrak{s u}(2)$ of $S U(2)$ given by

$$
X_{1}=\left(\begin{array}{cc}
i & 0 \\
0 & -i
\end{array}\right), \quad X_{2}=\left(\begin{array}{cc}
0 & 1 \\
-1 & 0
\end{array}\right), \quad X_{3}=\left(\begin{array}{cc}
0 & i \\
i & 0
\end{array}\right) .
$$

Then

$$
\left[X_{1}, X_{2}\right]=2 X_{3}, \quad\left[X_{2}, X_{3}\right]=2 X_{1}, \quad\left[X_{3}, X_{1}\right]=2 X_{2} .
$$

The one-parameter family $\left\{g_{\varepsilon}: \varepsilon>0\right\}$ of left-invariant Riemannian metrics on $\mathbb{S}^{3}=$ $S U(2)$ given at the identity, with respect to the basis of left-invariant vector fields $X_{1}, X_{2}, X_{3}$, by

$$
g_{\varepsilon}=\left(\begin{array}{ccc}
\varepsilon & 0 & 0 \\
0 & 1 & 0 \\
0 & 0 & 1
\end{array}\right),
$$

are called the Berger metrics on $\mathbb{S}^{3}$; if $\varepsilon=1$ we have the canonical (bi-invariant) metric. The Berger spheres are the simply connected complete Riemannian manifolds $\mathbb{S}_{\varepsilon}^{3}=$ $\left(\mathbb{S}^{3}, g_{\varepsilon}\right), \varepsilon>0$.

The Levi-Civita connection of $g_{\varepsilon}$ is given by

$$
2 g_{\varepsilon}\left(\nabla_{X} Y, Z\right)=g_{\varepsilon}([X, Y], Z)-g_{\varepsilon}([Y, Z], X)+g_{\varepsilon}([Z, X], Y),
$$

for all $X, Y, Z \in \mathfrak{s u}(2)$. So, the covariant derivatives between generators are given by $\nabla_{X_{i}} X_{i}=0$ and

$$
\begin{array}{lll} 
& \nabla_{X_{1}} X_{2}=(2-\varepsilon) X_{3}, & \nabla_{X_{1}} X_{3}=(\varepsilon-2) X_{2} \\
\nabla_{X_{2}} X_{1}=-\varepsilon X_{3}, & & \nabla_{X_{2}} X_{3}=X_{1}, \\
\nabla_{X_{3}} X_{1}=\varepsilon X_{2}, \quad \nabla_{X_{3}} X_{2}=-X_{1} . &
\end{array}
$$


The components of the curvature tensor field are given by

$$
\begin{array}{lll}
R_{X_{1} X_{2}} X_{1}=\varepsilon^{2} X_{2}, & R_{X_{1} X_{2} X_{2}=-\varepsilon X_{1},} & R_{X_{1} X_{2} X_{3}}=0 \\
R_{X_{1} X_{3} X_{1}}=\varepsilon^{2} X_{3}, & R_{X_{1} X_{3} X_{2}=0,} & R_{X_{1} X_{3} X_{3}}=-\varepsilon X_{1}, \\
R_{X_{2} X_{3} X_{1}}=0, & R_{X_{2} X_{3} X_{2}}=(4-3 \varepsilon) X_{3}, & R_{X_{2} X_{3} X_{3}}=(3 \varepsilon-4) X_{2} .
\end{array}
$$

\section{Homogeneous Riemannian structures on $\mathbb{S}_{\varepsilon}^{3}$}

If $S$ is a homogeneous Riemannian structure on $\mathbb{S}_{\varepsilon}^{3}$ and $\widetilde{\nabla}=\nabla-S$, the condition $\widetilde{\nabla} g=0$ in (2.1) is equivalent to $S_{X Y Z}+S_{X Z Y}=0$ for all $X, Y, Z \in \mathfrak{s u}(2)$. Then, if $\left\{\alpha^{1}, \alpha^{2}, \alpha^{3}\right\}$ is the basis of left-invariant forms dual to $X_{1}, X_{2}, X_{3}$, the tensor field $S$ of type $(0,3)$ can be written as

$$
S=\rho \otimes\left(\alpha^{1} \wedge \alpha^{2}\right)+\sigma \otimes\left(\alpha^{1} \wedge \alpha^{3}\right)+\tau \otimes\left(\alpha^{2} \wedge \alpha^{3}\right),
$$

where

$$
\rho(Z)=S_{Z X_{1} X_{2}}, \quad \sigma(Z)=S_{Z X_{1} X_{3}}, \quad \tau(Z)=S_{Z X_{2} X_{3}},
$$

for each vector field $Z$ on $\mathbb{S}^{3}$.

Moreover, the condition $\dot{\widetilde{\nabla}} R=0$ is equivalent to

$$
\left(\nabla_{Z} R\right)_{X Y V W}=-R_{S_{Z} X Y V W}-R_{X S_{Z} Y V W}-R_{X Y S_{Z} V W}-R_{X Y V S_{Z} W}
$$

for all $Z, X, Y, V, W \in \mathfrak{s u}(2)$. Replacing $(X, Y, V, W)$ in (3.3) by $\left(X_{1}, X_{3}, X_{2}, X_{3}\right)$ and $\left(X_{1}, X_{2}, X_{2}, X_{3}\right)$, respectively, we obtain that

$$
(\varepsilon-1) \rho=\varepsilon(\varepsilon-1) \alpha^{3}, \quad(\varepsilon-1) \sigma=-\varepsilon(\varepsilon-1) \alpha^{2} .
$$

It is easy to see that the condition $\widetilde{\nabla} R=0$ holds if and only if the equations (3.4) are satisfied. In the case of the canonical metric $(\varepsilon=1)$ this condition holds automatically.

In order to determine the conditions for the 1 -forms $\rho, \sigma, \tau$ such that $\widetilde{\nabla} S=0$, we first compute the connections forms $\widetilde{\omega}_{i j}$ of $\widetilde{\nabla}$, defined by $\widetilde{\nabla}_{Z} X_{j}=\sum_{i=1}^{3} \widetilde{\omega}_{i j}(Z) X_{i}$. They are given by $\widetilde{\omega}_{i i}=0$ and

$$
\begin{array}{lll} 
& \widetilde{\omega}_{21}=\varepsilon \alpha^{3}-\rho, \quad & \widetilde{\omega}_{31}=-\varepsilon \alpha^{2}-\sigma, \\
\widetilde{\omega}_{12}=-\alpha^{3}+\frac{1}{\varepsilon} \rho, & & \widetilde{\omega}_{32}=(2-\varepsilon) \alpha^{1}-\tau, \\
\widetilde{\omega}_{13}=\alpha^{2}+\frac{1}{\varepsilon} \sigma, & \widetilde{\omega}_{23}=(\varepsilon-2) \alpha^{1}+\tau . &
\end{array}
$$

Since $S_{Z X X}=0$, by using these last equations, from (3.2) we obtain that

$$
\begin{aligned}
& \left(\widetilde{\nabla}_{Z} S\right)_{V X_{1} X_{2}}=\left(\widetilde{\nabla}_{Z} \rho\right)(V)-\left(\varepsilon \alpha^{2}+\sigma\right)(Z) \tau(V)+\left((\varepsilon-2) \alpha^{1}+\tau\right)(Z) \sigma(V), \\
& \left(\widetilde{\nabla}_{Z} S\right)_{V X_{1} X_{3}}=\left(\widetilde{\nabla}_{Z} \sigma\right)(V)-\left(\varepsilon \alpha^{3}-\rho\right)(Z) \tau(V)+\left((2-\varepsilon) \alpha^{1}-\tau\right)(Z) \rho(V), \\
& \left(\widetilde{\nabla}_{Z} S\right)_{V X_{2} X_{3}}=\left(\widetilde{\nabla}_{Z} \tau\right)(V)+\left(\alpha^{3}-\frac{1}{\varepsilon} \rho\right)(Z) \sigma(V)+\left(\alpha^{2}+\frac{1}{\varepsilon} \sigma\right)(Z) \rho(V) .
\end{aligned}
$$

In particular, if $\varepsilon=1$ we have 
Theorem 3.1. The homogeneous Riemannian structures on the sphere $\mathbb{S}^{3}$ with the canonical metric are given by (3.1), where $\rho, \sigma$ and $\tau$ are differential 1-forms on $\mathbb{S}^{3}$ satisfying

$$
\begin{aligned}
& \widetilde{\nabla} \rho=\left(\alpha^{1}-\tau\right) \otimes \sigma+\left(\alpha^{2}+\sigma\right) \otimes \tau, \\
& \widetilde{\nabla} \sigma=\left(\alpha^{3}-\rho\right) \otimes \tau-\left(\alpha^{1}-\tau\right) \otimes \rho, \\
& \widetilde{\nabla} \tau=-\left(\alpha^{2}+\sigma\right) \otimes \rho-\left(\alpha^{3}-\rho\right) \otimes \sigma .
\end{aligned}
$$

Suppose now that $\varepsilon \neq 1$. By (3.4), the condition $\widetilde{\nabla} R=0$ is equivalent to $\rho=\varepsilon \alpha^{3}$ and $\sigma=-\varepsilon \alpha^{2}$. Then equations (3.5) reduce to

$$
\widetilde{\omega}_{23}=(\varepsilon-2) \alpha^{1}+\tau=-\widetilde{\omega}_{32}, \quad \widetilde{\omega}_{i j}=0 \text { in other case. }
$$

Since $\left(\widetilde{\nabla}_{Z} \alpha^{i}\right)\left(X_{j}\right)=-\widetilde{\omega}_{i j}(Z)$, by $(3.9)$ we have

$$
\widetilde{\nabla}_{Z} \alpha^{1}=0, \quad \widetilde{\nabla}_{Z} \alpha^{2}=\left((2-\varepsilon) \alpha^{1}-\tau\right)(Z) \alpha^{3}, \quad \widetilde{\nabla}_{Z} \alpha^{3}=\left((\varepsilon-2) \alpha^{1}+\tau\right)(Z) \alpha^{2},
$$

and by (3.6), (3.7) and (3.10), we have $\left(\widetilde{\nabla}_{Z} S\right)_{V X_{1} X_{2}}=\left(\widetilde{\nabla}_{Z} S\right)_{V X_{1} X_{3}}=0$, and hence, by (3.8), $\widetilde{\nabla} S=0$ if and only if $\widetilde{\nabla} \tau=0$. Now, if we put $\tau=f_{1} \alpha^{1}+f_{2} \alpha^{2}+f_{3} \alpha^{3}$, by using (3.10), the equation $\widetilde{\nabla} \tau=0$ is equivalent to the equations

$$
Z\left(f_{1}\right)=0, \quad Z\left(f_{2}\right)+f_{3}\left((\varepsilon-2) \alpha^{1}+\tau\right)(Z)=0, \quad Z\left(f_{3}\right)+f_{2}\left((2-\varepsilon) \alpha^{1}-\tau\right)(Z)=0,
$$

for every vector field $Z$ on $\mathbb{S}^{3}$. Then $f_{1}$ is a constant. Replacing $Z$ by $X_{2}$ and $X_{3}$ in each one of the last two equations above and using the structure equations (2.6), we obtain that $f_{2}=f_{3}=0$. So, $\tau$ is a scalar multiple of $\alpha^{1}$, and we conclude

Theorem 3.2. For $\varepsilon \neq 1$, the homogeneous Riemannian structures on the Berger sphere $\mathbb{S}_{\varepsilon}^{3}$ are given by

$$
S_{\varepsilon, t}=\varepsilon \alpha^{3} \otimes\left(\alpha^{1} \wedge \alpha^{2}\right)-\varepsilon \alpha^{2} \otimes\left(\alpha^{1} \wedge \alpha^{3}\right)+t \alpha^{1} \otimes\left(\alpha^{2} \wedge \alpha^{3}\right), \quad t \in \mathbb{R} .
$$

As a consequence, the components of the (1,2)-tensor field corresponding to $S=S_{\varepsilon, t}$ in (3.11) are given by

$$
\begin{aligned}
& S_{X_{1}} X_{1}=0, \quad S_{X_{1}} X_{2}=t X_{3}, \quad S_{X_{1}} X_{3}=-t X_{2}, \\
& S_{X_{2}} X_{1}=-\varepsilon X_{3}, \quad S_{X_{2}} X_{2}=0, \quad S_{X_{2}} X_{3}=X_{1}, \\
& S_{X_{3}} X_{1}=\varepsilon X_{2}, \quad S_{X_{3}} X_{2}=-X_{1}, \quad S_{X_{3}} X_{3}=0 .
\end{aligned}
$$

Remark 3.3. For each $p \in \mathbb{S}^{3}$, let $c_{12}(S)_{p}$ be the map from the tangent space $T_{p}\left(\mathbb{S}^{3}\right)$ to its dual given by $c_{12}(S)_{p}(Z)=\sum_{i=1}^{3} S_{e_{i} e_{i} Z}$, where $\left\{e_{i}\right\}$ is an orthonormal basis of $T_{p}\left(\mathbb{S}^{3}\right)$. If we choose $e_{1}=\sqrt{\varepsilon}^{-1} X_{1 \mid p}, e_{2}=X_{2 \mid p}, e_{3}=X_{3 \mid p}$, we see that $c_{12}(S)_{p}$ vanishes for every $S=S_{\varepsilon, t}$. According to Tricerri-Vanhecke's classification of homogeneous Riemannian structures in [15], this implies that each $S=S_{\varepsilon, t}$ is of type $\mathcal{T}_{2} \oplus \mathcal{T}_{3}$. Moreover, if $t=\varepsilon$, we have $S_{X} Y+S_{Y} X=0$, then $S_{\varepsilon, \varepsilon}$ is of type $\mathcal{T}_{3}$, which means that $\mathbb{S}_{\varepsilon}^{3}$ is a naturally reductive Riemannian space. If $t=-2 \varepsilon$, we have that each cyclic sum $\mathfrak{S}_{X Y Z} S_{X Y Z}$ vanishes, and hence $S_{\varepsilon,-2 \varepsilon}$ is of type $\mathcal{T}_{2}$, which may be also expressed by saying that $\mathbb{S}_{\varepsilon}^{3}$ is a cotorsionless manifold (see [7]). 
Remark 3.4. If $\varepsilon=1$, particular solutions $(\rho, \sigma, \tau)$ of equations in Theorem 3.1 are $\left(\alpha^{3},-\alpha^{2}, t \alpha^{1}\right),\left(\alpha^{3},-t \alpha^{2}, \alpha^{1}\right),\left(t \alpha^{3},-\alpha^{2}, \alpha^{1}\right)$, and $\left(t \alpha^{3},-t \alpha^{2}, t \alpha^{1}\right), t \in \mathbb{R}$. They give rise to four one-parameter families of homogeneous Riemannian structures on the standard sphere $\mathbb{S}^{3}$. The first of them is given by (3.11). If $t=0$ in the fourth family we get the solution $S=0$, what is equivalent to say that $\nabla R=0$ and it expresses the well-known fact that the standard sphere is a Riemannian symmetric space.

\section{Reductive decompositions and isometric actions on Berger spheres}

We shall determine the reductive decompositions associated to the homogeneous Riemannian structures on the Berger spheres $\mathbb{S}_{\varepsilon}^{3}$ given by $(3.11)$. We fix the point $o=(1,0) \in$ $\mathbb{S}^{3} \subset \mathbb{C}^{2}$, which corresponds to the identity matrix $I \in S U(2)$ and put $\widetilde{\mathfrak{m}}=T_{o}\left(\mathbb{S}^{3}\right)=$ $T_{I}(S U(2))=\mathfrak{s u}(2)$.

If $S=S_{\varepsilon, t}$ is the homogeneous Riemannian structure defined by (3.11) then the connection $\widetilde{\nabla}=\widetilde{\nabla}_{\varepsilon, t}=\nabla-S$ is given, with respect to the basis $\left\{X_{1}, X_{2}, X_{3}\right\}$ of $\mathfrak{s u}(2)$, by

$$
\widetilde{\nabla}_{X_{1}} X_{2}=(2-\varepsilon-t) X_{3}, \quad \widetilde{\nabla}_{X_{1}} X_{3}=(\varepsilon-2+t) X_{2},
$$

with the rest vanishing, and the components of the curvature tensor are $\widetilde{R}_{X_{1} X_{2}}=$ $\widetilde{R}_{X_{1} X_{3}}=0$ and $\widetilde{R}_{X_{2} X_{3}}=2(2-\varepsilon-t)\left(\alpha^{2} \otimes X_{3}-\alpha^{3} \otimes X_{2}\right)$. The holonomy algebra $\tilde{\mathfrak{h}}=\tilde{\mathfrak{h}}_{\varepsilon, t}$ of $\widetilde{\nabla}$ is the Lie algebra of antisymmetric endomorphisms of $\widetilde{\mathfrak{m}}=\mathfrak{s u}(2)$ generated by the curvature operators $\widetilde{R}_{X_{i} X_{j}}$, and the reductive pair associated to the homogeneous Riemannian structure $S=S_{\varepsilon, t}$ is $(\tilde{\mathfrak{g}}, \tilde{\mathfrak{h}})$, where $\tilde{\mathfrak{g}}=\tilde{\mathfrak{g}}_{\varepsilon, t}=\tilde{\mathfrak{h}} \oplus \widetilde{\mathfrak{m}}$ is a Lie algebra with the structure equations defined by $(2.4)$.

If $t=2-\varepsilon$, the holonomy algebra $\tilde{\mathfrak{h}}$ of $\widetilde{\nabla}$ is trivial and the reductive decomposition associated to the homogeneous Riemannian structure $S=S_{\varepsilon, 2-\varepsilon}$ is $\tilde{\mathfrak{g}}=\{0\} \oplus \mathfrak{s u}(2)$ with the structure equations (2.6). Then $\tilde{\mathfrak{g}}_{\varepsilon, 2-\varepsilon}=\mathfrak{s u}(2)$ and we have that the isometry group associated to $S=S_{\varepsilon, 2-\varepsilon}$ is $S U(2)$, acting on itself by left translations.

Suppose now that $t \neq 2-\varepsilon$. We put $a_{t}=(2-\varepsilon-t) / 2, b_{t}=(\varepsilon+t) / 2=1-a_{t}$. Then $U=\left(1 / 2 a_{t}\right) \widetilde{R}_{X_{2} X_{3}}=2\left(\alpha^{2} \otimes X_{3}-\alpha^{3} \otimes X_{2}\right)$ generates the holonomy algebra $\tilde{\mathfrak{h}}=\tilde{\mathfrak{h}}_{\varepsilon, t}$ of $\widetilde{\nabla}=\widetilde{\nabla}_{\varepsilon, t}$ and the reductive decomposition associated to the homogeneous Riemannian structure $S=S_{\varepsilon, t}$ is $\tilde{\mathfrak{g}}_{\varepsilon, t}=\tilde{\mathfrak{h}}_{\varepsilon, t} \oplus \mathfrak{s u}(2)=\left\langle\left\{U, X_{1}, X_{2}, X_{3}\right\}\right\rangle$, with the structure equations, by (2.4), given by

$$
\begin{array}{lll}
{\left[X_{1}, X_{2}\right]=2 b_{t} X_{3},} & {\left[X_{2}, X_{3}\right]=2 a_{t} U+2 X_{1},} & {\left[X_{3}, X_{1}\right]=2 b_{t} X_{2},} \\
{\left[U, X_{1}\right]=0,} & {\left[U, X_{2}\right]=2 X_{3},} & {\left[U, X_{3}\right]=-2 X_{2} .}
\end{array}
$$

If we put $\hat{U}=b_{t} U-X_{1}, \hat{X}_{1}=a_{t} U+X_{1}, \hat{X}_{2}=X_{2}, \hat{X}_{3}=X_{3}$, then

$$
\left[\hat{X}_{1}, \hat{X}_{2}\right]=2 \hat{X}_{3}, \quad\left[\hat{X}_{2}, \hat{X}_{3}\right]=2 \hat{X}_{1}, \quad\left[\hat{X}_{3}, \hat{X}_{1}\right]=2 \hat{X}_{2}, \quad\left[\hat{U}, \hat{X}_{j}\right]=0,(1 \leqslant j \leqslant 3),
$$

so $\tilde{\mathfrak{g}}_{\varepsilon, t}$ is the direct product of the Lie algebra $\mathfrak{r}=\langle\{\hat{U}\}\rangle$ of $\mathbb{R}$ and $\mathfrak{s u}(2)=\left\langle\left\{\hat{X}_{1}, \hat{X}_{2}, \hat{X}_{3}\right\}\right\rangle$. The corresponding connected simply connected Lie group is $\widetilde{G}=\mathbb{R} \times S U(2)$. 

by

Now, $\widetilde{G}=\mathbb{R} \times S U(2)$ acts transitively and almost effectively on each Berger sphere

$$
((s, g), p) \longmapsto g p\left(\begin{array}{cc}
e^{-i s} & 0 \\
0 & e^{i s}
\end{array}\right) .
$$

The isotropy group at the point $o=I \in \mathbb{S}^{3}=S U(2)$ is $\widetilde{H}=\left\{\left(s,\left(\begin{array}{cc}e^{i s} & 0 \\ 0 & e^{-i s}\end{array}\right)\right): s \in \mathbb{R}\right\}$. Let $\psi: \widetilde{G} \rightarrow U(2)$ be the covering homomorphism defined by $\psi(s, g)=e^{-i s} g$. It induces a 2-fold covering homomorphism $\mathbb{S}^{1} \times S U(2) \rightarrow U(2)$. The normal subgroup $N=\{(2 k \pi, I): k \in \mathbb{Z}\}$ of $\widetilde{G}$ acts trivially on $S U(2)$ and the action (4.2) induces a transitive and almost effective isometric action of $\widetilde{G} / N \cong \mathbb{S}^{1} \times S U(2)$ on $\mathbb{S}_{\varepsilon}^{3}$. The set $\Gamma$ of all the elements of $\widetilde{G}$ which act trivially on $\mathbb{S}^{3}$ is the discrete normal subgroup of $\widetilde{G}$ given by $\Gamma=\left\{\left(k \pi,(-1)^{k} I\right): k \in \mathbb{Z}\right\}=\operatorname{ker} \psi \subset \widetilde{H}$ and $G=\widetilde{G} / \Gamma \cong U(2)$ acts transitively and effectively on $\mathbb{S}_{\varepsilon}^{3}$ as a group of isometries. Moreover, $H=\widetilde{H} / \Gamma \cong \psi(H)=\{1\} \times U(1) \subset U(2)$ is the isotropy group at $o=(1,0) \equiv I$ of the action of $U(2)$ induced by (4.2).

We will show that these actions define reductive decompositions whose canonical connection, given by (2.3), defines the homogeneous Riemannian structures in (3.11). The Lie algebra of the groups $\widetilde{G}, \widetilde{G} / N$, and $\widetilde{G} / \Gamma$ are isomorphic to the direct product Lie algebra $\mathfrak{g}=\mathbb{R} \times \mathfrak{s u}(2)$. We consider the basis $\left\{B_{0}, B_{1}, B_{2}, B_{3}\right\}$ of $\mathfrak{g}$ given by $B_{0}=(D, 0)$, $B_{1}=\left(0, X_{1}\right), B_{2}=\left(0, X_{2}\right), B_{3}=\left(0, X_{3}\right)$, where $D$ is the canonical base vector of $\mathbb{R}=T_{0}(\mathbb{R})$ and $X_{1}, X_{2}, X_{3} \in \mathfrak{s u}(2)$ are again given by (2.5). Each one-parameter group $\left\{\exp s B_{j}\right\}$ generates a Killing vector field $B_{j}^{*}(1 \leqslant j \leqslant 4)$ on the sphere: $B_{0}^{*}=-X_{1}$ is left-invariant and, for $1 \leqslant j \leqslant 3, B_{j}^{*}$ is the right-invariant vector field on $S U(2)$ defined by $X_{j \mid I}$. On the other hand, if $U_{0}=B_{0}+B_{1}$ and we consider the exponential $\operatorname{map} \exp : \mathfrak{g} \rightarrow \widetilde{G}=\mathbb{R} \times S U(2)$, then $\left\{\exp s U_{0}: s \in \mathbb{R}\right\}=\widetilde{H}$. We put $\mathfrak{h}=\left\langle\left\{U_{0}\right\}\right\rangle$, $E_{t}=-a_{t} B_{0}+b_{t} B_{1}$, and $\mathfrak{m}=\left\langle\left\{E_{t}, B_{2}, B_{3}\right\}\right\rangle$; we have $\left[U_{0}, E_{t}\right]=0,\left[U_{0}, B_{2}\right]=2 B_{3}$, $\left[U_{0}, B_{3}\right]=-2 B_{2}$, and so $\mathfrak{g}=\mathfrak{h} \oplus \mathfrak{m}$ is a reductive decomposition. The isomorphism $\mu: \mathfrak{m} \rightarrow T_{o}\left(\mathbb{S}^{3}\right)=T_{I}(S U(2)) \equiv \mathfrak{s u}(2)$ in $(2.2)$ verifies $\mu\left(E_{t}\right)=\left(a_{t}+b_{t}\right) X_{1 \mid I} \equiv X_{1}$, $\mu\left(B_{2}\right)=X_{2 \mid I} \equiv X_{2}$, and $\mu\left(B_{3}\right)=X_{3 \mid I} \equiv X_{3}$. Moreover, $\left[E_{t}, B_{2}\right]=2 b_{t} B_{3},\left[E_{t}, B_{3}\right]=$ $2 a_{t} U_{0}+2 E_{t},\left[E_{t}, B_{3}\right]=-2 b_{t} B_{2}$, and by $(2.3)$, we get

$$
\begin{array}{lll}
\left(\widetilde{\nabla}_{E_{t}^{*}} E_{t}^{*}\right)_{I}=0, & \left(\widetilde{\nabla}_{E_{t}^{*}} B_{2}^{*}\right)_{I}=-2 b_{t} X_{3 \mid I}, & \left(\widetilde{\nabla}_{E_{t}^{*}} B_{3}^{*}\right)_{I}=2 b_{t} X_{2 \mid I}, \\
\left(\widetilde{\nabla}_{B_{2}^{*}} E_{t}^{*}\right)_{I}=2 b_{t} X_{3 \mid I}, & \left(\widetilde{\nabla}_{B_{2}^{*}} B_{2}^{*}\right)_{I}=0, & \left(\widetilde{\nabla}_{B_{2}^{*}} B_{3}^{*}\right)_{I}=-2 X_{1 \mid I}, \\
\left(\widetilde{\nabla}_{B_{3}^{*}} E_{t}^{*}\right)_{I}=-2 b_{t} X_{2 \mid I}, & \left(\widetilde{\nabla}_{B_{3}^{*}} B_{2}^{*}\right)_{I}=2 X_{1 \mid I}, & \left(\widetilde{\nabla}_{B_{3}^{*}} B_{3}^{*}\right)_{I}=0 .
\end{array}
$$

For each $p=(z, w) \equiv\left(\begin{array}{cc}z & w \\ -\bar{w} & \bar{z}\end{array}\right) \in \mathbb{S}^{3}=S U(2)$, we can write the left-invariant vector fields $X_{1}, X_{2}, X_{3}$ in terms of the fundamental vector fields $B_{1}^{*}, B_{2}^{*}, B_{3}^{*}$ as

$$
\begin{aligned}
& X_{1 \mid p}=\left(|z|^{2}-|w|^{2}\right) B_{1 \mid p}^{*}+2 \operatorname{Im}(z w) B_{2 \mid p}^{*}-2 \operatorname{Re}(z w) B_{3 \mid p}^{*} \\
& X_{2 \mid p}=2 \operatorname{Im}(z \bar{w}) B_{1 \mid p}^{*}+\operatorname{Re}\left(z^{2}+w^{2}\right) B_{2 \mid p}^{*}+2 \operatorname{Im}\left(z^{2}+w^{2}\right) B_{3 \mid p}^{*}, \\
& X_{3 \mid p}=2 \operatorname{Re}(z \bar{w}) B_{1 \mid p}^{*}+\operatorname{Im}\left(w^{2}-z^{2}\right) B_{2 \mid p}^{*}+2 \operatorname{Re}\left(z^{2}-w^{2}\right) B_{3 \mid p}^{*} .
\end{aligned}
$$


By using (4.4) and (4.3), some computations show that

$$
\begin{aligned}
& \left(\widetilde{\nabla}_{X_{1}} X_{2}\right)_{I}=\left(\widetilde{\nabla}_{E_{t}^{*}} B_{2}^{*}\right)_{I}+2 B_{3 \mid I}^{*}=\left(2-2 b_{t}\right) X_{3 \mid I}=2 a_{t} X_{3 \mid I}, \\
& \left(\widetilde{\nabla}_{X_{1}} X_{3}\right)_{I}=-2 B_{2 \mid I}^{*}+\left(\widetilde{\nabla}_{E_{t}^{*}} B_{3}^{*}\right)_{I}=\left(-2+2 b_{t}\right) X_{2 \mid I}=-2 a_{t} X_{2 \mid I},
\end{aligned}
$$

and the remaining $\left(\widetilde{\nabla}_{X_{j}} X_{k}\right)_{I}$ vanish. As a consequence, using (2.7), we see that the homogeneous Riemannian structure $S=\nabla-\widetilde{\nabla}$ is given by equations (3.12). Now, if $\psi_{*}: \mathfrak{g}=\mathbb{R} \times \mathfrak{s u}(2) \rightarrow \mathfrak{u}(2)$ is the Lie algebra isomorphism induced by the covering homomorphism $\psi$, then $\psi_{*}\left(U_{0}\right)=\left(\begin{array}{cc}0 & 0 \\ 0 & -2 i\end{array}\right), \psi_{*}\left(E_{t}\right)=\left(\begin{array}{cc}i & 0 \\ 0 & (1-\varepsilon-t) i\end{array}\right), \psi_{*}\left(B_{2}\right)=\left(\begin{array}{cc}0 & 1 \\ -1 & 0\end{array}\right)$, $\psi_{*}\left(B_{3}\right)=\left(\begin{array}{cc}0 & i \\ i & 0\end{array}\right)$, and $\mathfrak{u}(2)=\psi_{*}(\mathfrak{h}) \oplus \psi_{*}(\mathfrak{m})$ is the reductive decomposition of $\mathbb{S}_{\varepsilon}^{3}=$ $U(2) / U(1)$ associated to $S_{\varepsilon, t}$. We conclude

Theorem 4.1. Let $S=S_{\varepsilon, t}$ be the homogeneous Riemannian structure on the Berger sphere $\mathbb{S}_{\varepsilon}^{3}$ defined by (3.12). If $t=2-\varepsilon$, the associated reductive decomposition is trivial and the corresponding group of isometries is $S U(2)$ acting on itself by left translations.

The group $\mathbb{S}^{1} \times S U(2)$ acts transitively and almost effectively on $\mathbb{S}^{3}=S U(2)$ by

$$
\left(\left(e^{i s}, g\right), p\right) \longmapsto g p\left(\begin{array}{cc}
e^{-i s} & 0 \\
0 & e^{i s}
\end{array}\right),
$$

and induces a transitive and effective action of $U(2)$ on $\mathbb{S}_{\varepsilon}^{3}$ as a group of isometries, whose reductive decomposition

$$
\mathfrak{u}(2)=\left\langle\left\{\left(\begin{array}{ll}
0 & 0 \\
0 & i
\end{array}\right)\right\}\right\rangle \bigoplus\left\langle\left\{\left(\begin{array}{cc}
i & 0 \\
0 & (1-\varepsilon-t) i
\end{array}\right),\left(\begin{array}{cc}
1 & 0 \\
0 & -1
\end{array}\right),\left(\begin{array}{ll}
0 & i \\
i & 0
\end{array}\right)\right\}\right\rangle
$$

is the associated one to the homogeneous Riemannian structure $S$ if $t \neq 2-\varepsilon$; if $t=\varepsilon$ it is a naturally reductive decomposition.

Remark 4.2. In the case of the standard sphere $(\varepsilon=1), S=0$ is a homogeneous Riemannian structure, the associated canonical connection is $\widetilde{\nabla}=\nabla$, the holonomy algebra is $\tilde{\mathfrak{h}} \cong \mathfrak{s o}(3)$, the corresponding reductive decomposition is $\tilde{\mathfrak{g}} \cong \mathfrak{s o}(4)=\mathfrak{s o}(3) \oplus \widetilde{\mathfrak{m}}$, and defines the representation of $\mathbb{S}^{3}$ as symmetric space $\mathbb{S}^{3}=S O(4) / S O(3)$. As we noticed in Remark 3.4, different families of homogeneous Riemannian structures on the standard sphere $\mathbb{S}^{3}$ are also given by putting $(\rho, \sigma, \tau)=\left(\alpha^{3},-\alpha^{2}, t \alpha^{1}\right),\left(\alpha^{3},-t \alpha^{2}, \alpha^{1}\right)$ or $\left(t \alpha^{3},-\alpha^{2}, \alpha^{1}\right)$ in equation (3.1); if $t \neq 1$, all of them correspond to the isometric action of $U(2)$ on $\mathbb{S}^{3}$, with different reductive decompositions of $\mathbb{S}^{3}=U(2) / U(1)$; if $t=1$, all of them define the same homogeneous Riemannian structure $S=\alpha^{3} \otimes\left(\alpha^{1} \wedge \alpha^{2}\right)-\alpha^{2} \otimes$ $\left(\alpha^{1} \wedge \alpha^{3}\right)+\alpha^{1} \otimes\left(\alpha^{2} \wedge \alpha^{3}\right)$, which corresponds to the isometric action of $S U(2)$ on itself by left translations.

Remark 4.3. Tricerri and Vanhecke pointed out in [15] that given a homogeneous Riemannian manifold $(M, g)$ with a group $G$ of isometries, Ambrose-Singer's method determines a tensor field $S$. This determines conversely a group $G^{\prime}$ of isometries which is in general not isomorphic to $G$. A simple but interesting example is the Euclidean plane $\mathbb{R}^{2}$. From the connected group of isometries $G=S O(2) \cdot \mathbb{R}^{2}$ one obtains $S=0$. Now, the construction of $G^{\prime}$ starting from $S=0$ gives only the group of translations of 
$\mathbb{R}^{2}$, since $\mathbb{R}^{2}$ is flat. So an interesting problem is to understand for which spaces one has $G=G^{\prime}$ (see [15],[12]). The discussion in the present section shows that Berger spheres provide examples of such spaces.

\section{The Berger spheres as homogeneous almost contact metric manifolds}

An almost contact structure on a $(2 n+1)$-dimensional manifold $M$ is a triple $(\varphi, \xi, \eta)$, where $\varphi$ is a tensor field of type $(1,1), \xi$ a vector field (called the characteristic vector field) and $\eta$ a differential 1-form on $M$ such that

$$
\varphi^{2}=-\mathrm{id}+\eta \otimes \xi, \quad \eta(\xi)=1 .
$$

Then $\varphi \xi=0, \eta \circ \varphi=0$, and $\varphi$ has rank $2 n$. If $g$ is a Riemannian metric on $M$ such that $g(\varphi X, \varphi Y)=g(X, Y)-\eta(X) \eta(Y)$ for all vector fields $X$ and $Y$ on $M$ then $(\varphi, \xi, \eta, g)$ is said to be an almost contact metric structure on $M$ and $g$ is called a compatible metric; in this case, $g(X, \xi)=\eta(X)$ and $\xi$ has length 1 . If $d \eta(X, Y)=X \eta(Y)-Y \eta(X)-\eta([X, Y])=$ $2 g(X, \varphi Y)$, then $(\eta, g)$ is called a contact metric (or contact Riemannian) structure; in particular, $\eta \wedge(d \eta)^{n} \neq 0$, that is, $\eta$ is a contact form on $M$. If

$$
\left(\nabla_{X} \varphi\right) Y=\alpha(g(X, Y) \xi-\eta(Y) X),
$$

for a function $\alpha$ on $M$, then $(\varphi, \xi, \eta, g)$ is called $\alpha$-Sasakian, and the manifold $M$ with such a structure is an $\alpha$-Sasakian manifold. If $\alpha=1$, then it is Sasakian. Sasakian manifolds can also be characterized as normal contact metric manifolds and they are in some sense odd-dimensional analogues of Kähler manifolds (see Blair $[4,5]$ ).

If $(\varphi, \xi, \eta, g)$ is an almost contact metric structure on $M$ and $(M=G / H, g)$ is a homogeneous Riemannian manifold such that $\varphi$ is invariant under the action of $G$ then $(M, \varphi, \xi, \eta, g)$ is called a homogeneous almost contact metric manifold $([6,8]$, see also [11]). From the results of Kiričenko in [10] it follows that if $(M, g)$ is a connected, simply connected and complete Riemannian manifold and $(\varphi, \xi, \eta)$ is an almost contact structure on $M$ such that $g$ is a compatible metric, then $(M, \varphi, \xi, \eta, g)$ is a homogeneous almost contact metric manifold if and only if there exists a tensor field $S$ of type $(1,2)$ on $M$ satisfying (2.1) and the additional condition $\widetilde{\nabla} \varphi=0$, where $\widetilde{\nabla}=\nabla-S$; in this case, $\widetilde{\nabla} \xi=0, \widetilde{\nabla} \eta=0$, and $\xi$ and $\eta$ are also invariant under the action of the group ([6]). Such a homogeneous Riemannian structure $S$ is called a homogeneous almost contact metric structure on $(M, \varphi, \xi, \eta, g)$.

For each $\varepsilon>0$, we will define an almost contact structure $(\varphi, \xi, \eta)$ on the sphere $\mathbb{S}^{3}$ such that the Berger metric $g_{\varepsilon}$ may be compatible. In order to get that $\left(\varphi, \xi, \eta, g_{\varepsilon}\right)$ can be homogeneous, the characteristic vector field $\xi$ must be invariant by the isometries defined by the action of $S U(2)$ on itself, then $\xi$ must be left-invariant; and by (4.1), in order to have $\widetilde{\nabla} \xi=0$ for each homogeneous Riemannian structure $S_{\varepsilon, t}, t \neq 2-\varepsilon$, the vector field $\xi$ must be a scalar multiple of $X_{1}$; and it must have length 1 , then $\xi=\sqrt{\varepsilon}^{-1} X_{1}$; moreover, in order to obtain $g(X, \xi)=\eta(X)$ for each vector field $X$, the distribution 
defined by $\eta=0$ must be generated by the left-invariant vector fields $X_{2}, X_{3}$. We put

$$
\varphi=\alpha^{2} \otimes X_{3}-\alpha^{3} \otimes X_{2}, \quad \xi_{\varepsilon}=\frac{1}{\sqrt{\varepsilon}} X_{1}, \quad \eta_{\varepsilon}=\sqrt{\varepsilon} \alpha^{1} .
$$

Remark 5.1. Notice that $\eta_{\varepsilon} \wedge d \eta_{\varepsilon}=-2 \varepsilon \alpha^{1} \wedge \alpha^{2} \wedge \alpha^{3} \neq 0$, so $\eta_{\varepsilon}$ is a contact form on the 3 -sphere, and, moreover, $d \eta_{\varepsilon}(X, Y)=2 \sqrt{\varepsilon} g_{\varepsilon}(X, \varphi Y)$; in particular, $\left(\eta_{1}, g_{1}\right)$ is a contact Riemannian structure. The simply connected 3 -manifolds which admit homogeneous Riemannian contact structures (that is, those for which there exists a connected Lie group acting transitively as a group of isometries which leave the contact form invariant) have been classified by Perrone in [13].

The Levi-Civita connection $\nabla$ of $g_{\varepsilon}$ satisfies

$$
\left(\nabla_{X_{2}} \varphi\right) X_{1}=-\varepsilon X_{2}, \quad\left(\nabla_{X_{3}} \varphi\right) X_{1}=-\varepsilon X_{3}, \quad\left(\nabla_{X_{2}} \varphi\right) X_{2}=\left(\nabla_{X_{3}} \varphi\right) X_{3}=X_{1},
$$

and $\left(\nabla_{X_{i}} \varphi\right) X_{j}=0$ in the other cases. So, we have

$$
\left(\nabla_{X} \varphi\right) Y=\sqrt{\varepsilon}\left(g_{\varepsilon}(X, Y) \xi-\eta_{\varepsilon}(Y) X\right),
$$

for all vector fields $X$ and $Y$ on $\mathbb{S}^{3}$, hence $\left(\varphi, \xi_{\varepsilon}, \eta_{\varepsilon}, g_{\varepsilon}\right)$ is a $\sqrt{\varepsilon}$-Sasakian structure on $\mathbb{S}^{3}$.

If $\varepsilon \neq 1$, each homogeneous Riemannian structure $S=S_{\varepsilon, t}$ on $\mathbb{S}_{\varepsilon}^{3}$ is given by (3.11) and the canonical connection $\widetilde{\nabla}=\nabla-S$ satisfies (4.1). This implies $\widetilde{\nabla} \varphi=0$, and we have

Theorem 5.2. If $\varepsilon \neq 1$, all the homogeneous Riemannian structures on $\mathbb{S}_{\varepsilon}^{3}$ are homogeneous almost contact metric structures on the $\sqrt{\varepsilon}$-Sasakian manifold $\mathbb{S}^{3}\left(\varphi, \xi_{\varepsilon}, \eta_{\varepsilon}, g_{\varepsilon}\right)$.

Suppose now that $\varepsilon=1$. Each homogeneous Riemannian structure $S$ on the standard sphere $\mathbb{S}^{3}$ is given by (3.1), where $\rho, \sigma$, and $\tau$ are 1 -forms on $\mathbb{S}^{3}$ satisfying the equations in Theorem (3.1). If $\widetilde{\nabla}=\nabla-S$, then $\widetilde{\nabla}_{Z} X_{1}=\nabla_{Z} X_{1}-\rho(Z) X_{2}-\sigma(Z) X_{3}, \widetilde{\nabla}_{Z} X_{2}=$ $\nabla_{Z} X_{2}+\rho(Z) X_{1}-\tau(Z) X_{3}$, and $\widetilde{\nabla}_{Z} X_{3}=\nabla_{Z} X_{3}+\sigma(Z) X_{1}+\tau(Z) X_{2}$, and as a consequence we have that $\widetilde{\nabla} \varphi=0$ if and only if $\rho=\alpha^{3}$ and $\sigma=-\alpha^{2}$; if this is the case, the first and second equations in Theorem (3.1) are automatically satisfied and the third equation remains $\widetilde{\nabla} \tau=0$, which one gets it is equivalent to requiring that $\tau$ be a scalar multiple of $\alpha^{1}$ (such as in the case that $\varepsilon \neq 1$ in $\S 3$ ). Then we have

Theorem 5.3. The homogeneous almost contact metric structures on the Sasakian manifold $\mathbb{S}^{3}\left(\varphi, \xi_{1}, \eta_{1}, g_{1}\right)$ are given by

$$
S_{t}=\alpha^{3} \otimes\left(\alpha^{1} \wedge \alpha^{2}\right)-\alpha^{2} \otimes\left(\alpha^{1} \wedge \alpha^{3}\right)+t \alpha^{1} \otimes\left(\alpha^{2} \wedge \alpha^{3}\right), \quad t \in \mathbb{R} .
$$

If $t \neq 1$, the corresponding group of isometries leaving invariant the almost contact structure $\left(\varphi, \xi_{1}=X_{1}, \eta_{1}=\alpha^{1}\right)$ is $U(2)$ and the associated reductive decomposition is

$$
\mathfrak{u}(2)=\left\langle\left\{\left(\begin{array}{ll}
0 & 0 \\
0 & i
\end{array}\right)\right\}\right\rangle \bigoplus\left\langle\left\{\left(\begin{array}{cc}
i & 0 \\
0 & -t i
\end{array}\right),\left(\begin{array}{cc}
1 & 0 \\
0 & -1
\end{array}\right),\left(\begin{array}{ll}
0 & i \\
i & 0
\end{array}\right)\right\}\right\rangle .
$$

If $t=1$, the associated reductive decomposition is trivial and the corresponding group of isometries is $S U(2)$. 


\section{References}

[1] Ambrose, W. and Singer, I.M., On homogeneous Riemannian manifolds. Duke Math. J. 25, 647-669 (1958).

[2] Bérard-Bergery, L. and Bourguignon, J.P., Laplacians and Riemannian submersions with totally geodesic fibres. Illinois J. Math. 26, 181-200 (1982).

[3] Berger, M., Les variétés riemanniennes homogènes normales simplement connexes à courbure strictement positive. Ann. Scuola Norm. Sup. Pisa 15, 179-246 (1961).

[4] Blair, D.E., Contact manifolds in Riemannian geometry. Lecture Notes in Mathematics, 509. Springer-Verlag, Berlin-New York, 1976.

[5] Blair, D.E., Riemannian geometry of contact and symplectic manifolds. Progress in Mathematics, 203. Birkhäuser Boston, Inc., Boston, MA, 2002.

[6] Chinea, D. and González, J.C., An example of an almost cosymplectic homogeneous manifold. Differential geometry, Peñíscola 1985, 133-142, Lecture Notes in Mathematics, 1209, Springer-Verlag, Berlin, 1986.

[7] Gadea, P.M. and Oubiña, J.A., Reductive homogeneous pseudo-Riemannian manifolds. Monatsh. Math. 124, 17-34 (1997).

[8] GonzÁlez, J.C. and Chinea, D., Quasi-Sasakian homogeneous structures on the generalized Heisenberg group $H(p, 1)$. Proc. Amer. Math. Soc. 105, 173-184 (1989).

[9] Janssens, D. and VANHECKE, L., Almost contact structures and curvature tensors. Kōdai Math. J. 4, 1-27 (1981).

[10] KiRIČENKo, V.F., On homogeneous Riemannian spaces with invariant tensor structures. Soviet Math. Dokl. 21, 734-737 (1980).

[11] Koda, T. and Watanabe, Y., Homogeneous almost contact Riemannian manifolds and infinitesimal models. Boll. Un. Mat. Ital. B (7) 11-B, Suppl. fasc. 2, 11-24 (1997).

[12] Kowalski, O., Generalized symmetric spaces. Lecture Notes in Mathematics, 805. Springer-Verlag, Berlin-New York, 1980.

[13] Perrone, D., Homogeneous contact Riemannian three-manifolds. Illinois J. Math. 42, 243-256 (1998).

[14] Petersen, P., Riemannian Geometry, Springer-Verlag, New York, 1998.

[15] Tricerri, F. and Vanhecke, L., Homogeneous Structures on Riemannian Manifolds. London Math. Soc. Lect. Notes 83. Cambridge: Cambridge University Press. 1983.

[16] Urakawa, H., On the least positive eigenvalue of the Laplacian for compact group manifolds. J. Math. Soc. Japan 31, 209-226 (1979). 